\title{
Robust CLOS Guidance and Control: Part-1: System Modeling and Uncertainty Evaluation
}

\author{
A.N. Oda ${ }^{*}$, G.A. El-Sheikh ${ }^{\dagger}$, Y.Z. El-Halwagy ${ }^{*}$ and M. Al-Ashry ${ }^{*}$
}

\begin{abstract}
The great developments in applied mathematics and computational capabilities facilitate the design and implementation of robust control. In addition, the huge developments in nanotechnology and its availability in civilian level with less cost, size and weight attract many of the researchers allover the world towards embedded systems especially the embedded flight control. Among the real applications are the guided missiles especially the antitank guided missile systems which are commanded to the line of sight (CLOS) against ground and short range targets. The present work is concerned with improving the performance of an antitank guided missile system belonging to the first generation via robust synthesis of autopilot and guidance systems. The design and analysis necessitates somehow accurate model with different uncertainties (objective of Part-1 of the paper) for the system, a robust autopilot design (objective of Part-2 of the paper) and implementation via hardware in the loop (HIL) simulation (objective of Part-3 of the paper).
\end{abstract}

This part of the paper is devoted to the derivation of the system equations of motion clarifying different sources of uncertainty including thrust aging, anomalies in aerodynamic coefficients and derivatives and wind velocity effects. The solution of these equations is described in the form of modules programmed within the $\mathrm{C}++$ and MATLAB environments to form the baseline for subsequent design and analysis. The simulation is conducted with different engagement scenarios and different levels of uncertainty in thrust, aerodynamics and wind velocity. The simulation results are validated against reference data and verified for performance requirements including the time of flight, miss distance, control currents, normal acceleration and angles of attack. This investigation clarified the closeness of the designed model performance to the reference and its appropriateness for autopilot design and HIL simulation in the next parts of the paper.

Keywords: Command Guidance Systems, CLOS, Robust Control, Uncertainties

\section{Nomenclature}

$\mathbf{X}_{\mathbf{1}}, \mathbf{Y}_{\mathbf{1}}$, and $\mathbf{Z}_{\mathbf{1}}$
$\mathbf{X}_{\mathbf{g}}, \mathbf{Y}_{\mathbf{g}}$, and $\mathbf{Z}_{\mathbf{g}}$
$\mathbf{X}, \mathbf{Y}$, and $\mathbf{Z}$
$\mathbf{T}_{\mathbf{b g}}$
$\mathbf{T}_{\mathbf{v g}}$
$\mathbf{T}_{\mathbf{b g}}$
$\delta_{\mathbf{j p}}$ and $\delta_{\mathbf{j y}}$
$F_{T X_{1}}, F_{T Y 1}$, and $F_{T Z_{1}}$
$F_{A X}, F_{A Y}$, and $F_{A Z}$
$\mathbf{S}$
$\mathbf{Q}$

\author{
Vectors components along the board reference axes \\ Vectors components along the ground reference axes \\ Vectors components along the velocity reference axes \\ Transformation matrix from board to ground reference axes \\ Transformation matrix from velocity to ground reference axes \\ Transformation matrix from board to ground reference axes \\ Thrust jetivator angles in pitch and yaw planes \\ Thrust forces along the board reference axes \\ Drag, lateral, and lift forces along the velocity axes \\ Characteristic area \\ Dynamic pressure given by $\mathrm{q}=0.5 \rho(\mathrm{vm}) 2 \quad[\mathrm{Kg} / \mathrm{m} / \mathrm{sec} 2]$
}

\footnotetext{
* Egyptian Armed Forces, Egypt

${ }^{\dagger}$ Professor, , gaelsheikh@gmail.com, Tel. 02,01002682402
} 


$\boldsymbol{\rho}$
$\mathbf{V}_{\mathbf{M}}$
$\mathbf{C}_{\mathbf{x}}, \mathbf{C}_{\mathbf{y}}$, and $\mathbf{C}_{\mathbf{z}}$
$\mathbf{m}_{\mathbf{s}}$
$\bar{g}$
$\mathbf{m}_{\mathbf{o}}$
$\mathbf{m}_{\mathbf{s e c}}$
$\mathbf{M}$
$\mathbf{V}_{\mathbf{a}}$
$l_{T}$
$l_{T X}$
$l_{x}, l_{y}, l_{z}$
$m_{x_{1}}, m_{y_{1}}$, and $m_{z_{1}}$
$\omega_{x_{1}}, \omega_{y_{1}}$ and $\omega_{z_{1}}$
$\bar{J}$
$\Omega$
$\mathbf{I}_{\mathbf{X X}}, \mathbf{I}_{\mathbf{Y} \mathbf{Y}}$, and $\mathbf{I}_{\mathbf{Z Z}}$
$\boldsymbol{\alpha}$
$\boldsymbol{\beta}$
$\mathbf{U}, \mathbf{V}$, and $\mathbf{W}$
$\mathbf{U}_{\mathbf{d}}, \mathbf{V}_{\mathbf{d}}$, and $\mathbf{W}_{\mathbf{d}}$
$\mathbf{g}_{\mathbf{x}}, \mathbf{g}_{\mathbf{Y}}$, and $\mathbf{g}_{\mathbf{Z}}$
$\boldsymbol{\varepsilon}_{\mathbf{T}}$ and $\boldsymbol{\delta}_{\mathbf{T}}$
$\boldsymbol{\varepsilon}_{\mathbf{M}}$ and $\boldsymbol{\varepsilon}_{\mathbf{M}}$
$\boldsymbol{\Delta} \boldsymbol{\varepsilon}$ and $\boldsymbol{\Delta}_{\boldsymbol{\delta}}$
$\mathbf{R}_{\mathbf{m}}$ and $\mathbf{R}_{\mathbf{t}}$
$\boldsymbol{\theta}_{\mathbf{p}}$
$\boldsymbol{\psi}_{\mathbf{s}}$
$\boldsymbol{\varepsilon}_{\mathbf{1}}, \boldsymbol{\sigma}_{\mathbf{1}}$
$\mathbf{E}_{\mathbf{o}}$
$\boldsymbol{\theta}_{\mathbf{c}}$
$\boldsymbol{\theta}_{\mathbf{s}}$
$\boldsymbol{\mu} \mathbf{A}$
$\mathbf{N}$

Abbreviations

ACLOS

ADC

ATGM

BCS

CBR

C.G.

CLOS

C.P.

CRB

E-D

FOR-MON

FOV

FSM

GCS
Air density $\left[\mathrm{kg} / \mathrm{m}^{3}\right]$

Missile velocity

Dimension-less aerodynamic coefficients

Instantaneous total missile mass

Vector of gravity acceleration

Initial missile mass and

Burnt quantity of fuel or propellant per second

Mach number and given by $\mathrm{M}=\mathrm{v}_{\mathrm{m}} / \mathrm{v}_{\mathrm{a}}$

Sound velocity at missile position

Perpendicular distance between the missile C.G. and the action-point of lateral thrust forces

Perpendicular distance between longitudinal axis and thrust force line

Characteristic linear dimensions of missile

Dimensionless aerodynamic coefficients

Airframe-turn rates along board coordinate axes

Acceleration of missile

Angular velocity of VCS with respect to GCS

Moments of inertia components along the BCS

Angle of attack [angle of incidence] [Degree]

Sideslip angle [angle of drift] [Degree]

Velocities Along board coordinate axis

Derivative of velocities along board coordinate axis

Gravity acceleration along board coordinate axis

Elevation and azimuth angles of target

Elevation and azimuth angles of missile

LOS angular error

Missile and target range

Pitch demand

Angle between missile and LOS in yaw plane

LOS angular errors for the two planes expressed in meters

Temperature compensation term

Crest angle

Angle between sight forward axis and LOS in pitch plane

Micro ampere

Newton

Automatic commanded to line of sight

Aero Dynamic Coefficients

Anti Tank Guided Missile

Board coordinate system

Transformation matrix from board to reference coordinate System

Missile center of gravity

Commanded to line of sight

Center of pressure

Transformation matrix from reference to board Coordinate System

Electronic Driver

Force-Moment

Field of view

Flight simulation model

Ground coordinate system 


$\begin{array}{ll}\text { HIL } & \text { hardware in the loop } \\ \text { I/P } & \text { Input } \\ \text { IR } & \text { Infra-Red } \\ \text { LOS } & \text { Line of sight } \\ \text { MCLOS } & \text { Manual command to line of sight } \\ \text { PEC } & \text { Programmer electronic controller } \\ \text { PD } & \text { Proportional Derivative } \\ \text { PI } & \text { Proportional Integral } \\ \text { PID } & \text { Proportional Integral Derivative } \\ \text { RK4 } & \text { Runge- Kutta 4 } \\ \text { SACLOS } & \text { Semi-Automatic command to line of sight } \\ \text { 6-DOF } & \text { Six degrees of freedom } \\ \text { TF } & \text { Transfer function } \\ \text { TVC } & \text { Thrust vector control } \\ \text { VCS } & \text { Velocity coordinate system }\end{array}$

\section{Introduction}

Antitank guided missiles (ATGM) are command guidance systems launched against tanks and armored vehicles. These missiles are classified into three generations; the first generation in which both the target and missile are manually tracked using optical telescopes. The second generation in which the target is manually tracked using optical telescopes while the missile is automatically tracked by including an infrared sensor in the launcher with the telescope to detect the IR radiation from a source strapped on the rear part of the missile. Then the motion parameters are transferred automatically to signals applied to the guidance unit. The third generation is characterized by manual or automatic target tracking through optical telescopes, TV, laser or radio devices and the missile is automatically tracked as in the second generation. However, the guidance commands in this generation are transmitted to the missile through a remote link instead of wires. Note that this generation could be of the semi-active homing guidance in which guidance commands are generated onboard.In a command guidance system an operator or a computer at the control point solves the mission of interception on the basis of obtained coordinates for both target and missile and forms the command, according to the utilized or specified guidance method, for the control system on the missile, which changes its spatial position. A telescope or TV camera based on the parent platform tracks the target and the missile to yield tracking data to be sent to the system guidance computer. The guidance computer compares the two sets of tracking data (for target and missile) and extracts the appropriate corrections (guidance commands) according to the employed guidance method. Then, it applies these commands to the missile through a wire link during its flight. That is, the guidance of a missile is carried out either totally by the operator or partially by the interaction between human and electronic circuits constituting the guidance unit. According to these features, the antitank command guidance systems are divided into three main sub groups: manual command to line of sight (MCLOS), semi-automatic CLOS (SACLOS) and automatic CLOS (ACLOS) $[1,2,7,8,15,16]$. However, these literature did not manipulate the different sources of uncertainty corrupting the performance of such systems; one of the objectives in this work.

Using a command link imposes some limitations upon the guidance system such as data rate of transfer, loop delay and jamming. In addition, the ever-increasing role of armored forces in modern combat directs the designers and manufacturers towards increasing the tank capabilities. These capabilities include tank power and design improvement, armor production, maneuverability of tanks and jamming. These ever-increasing developments of tanks' capabilities necessitate the design of accurate guidance and control system for an antitank missile in presence of disturbance, measurement noise, and un-modelled dynamics. In addition, the underlying system has different sources of uncertainty including the forward loop gains, the thrust values due to aging, aerodynamic coefficients and derivatives due to lack of accurate wind tunnel tests, and expected wind velocities. These uncertainties lead, e.g., to anomalies in the missile flight behavior just after launch and yield to bring the missile 
down at lower values of thrust such that it impacts the ground. To overcome uncertainties and achieve the mission, this paper is devoted to derive an adequate nonlinear mathematical model representing the dynamical behaviour of the underlying missile for different flight phases and with uncertainties' quantification.

The evaluation of obtained mathematical model is carried out via simulation which is indispensable tool in the design and development and where the set of equations are programmed within MATLAB and $\mathrm{C}++$ environments. The input stimuli are launch conditions (pitch and yaw angles), target position, target motion and its trajectory characterization. The outputs are the missile flight data (thrust profile, speed, acceleration, range, angle of attack, incidence angle, jetivator deflections, flight time, miss distance, etc.) during the engagement. The obtained six degrees of freedom (6DOF) mathematical model consists of the equations describing kinematics, dynamics (weight, thrust, aerodynamic forces), command guidance generation system, instruments, compensation electronics, autopilot variables and estimates for the different sources of uncertainty. Using these models it is possible to draw contribution about the reliability of conceptual hardware design and hence to the requirement for the number of very expensive flight trials. In addition, it enables questions to be answered that it would not be possible to answer in any other way and consequently the system performance can be evaluated against futuristic targets [14].

\section{Missile Flight Modeling}

\subsection{Reference Frames and Transformation}

When formulating and solving problems of flying vehicles, coordinate systems and reference frames have to be considered for the description of the various dynamical parameters including position, velocity, acceleration, forces, and moments, Figs 1, 2, 3. The forces acting on the missile (flying vehicle) are weight, thrust, and aerodynamic forces. These forces have different mother frames of reference and consequently coordinates transformation from a frame to another is indispensable. This transformation is carried out using Euler's angles transformation method. The coordinates' transformation from body into the ground coordinate system using Euler's angles can be carried out using the following transformation matrix:

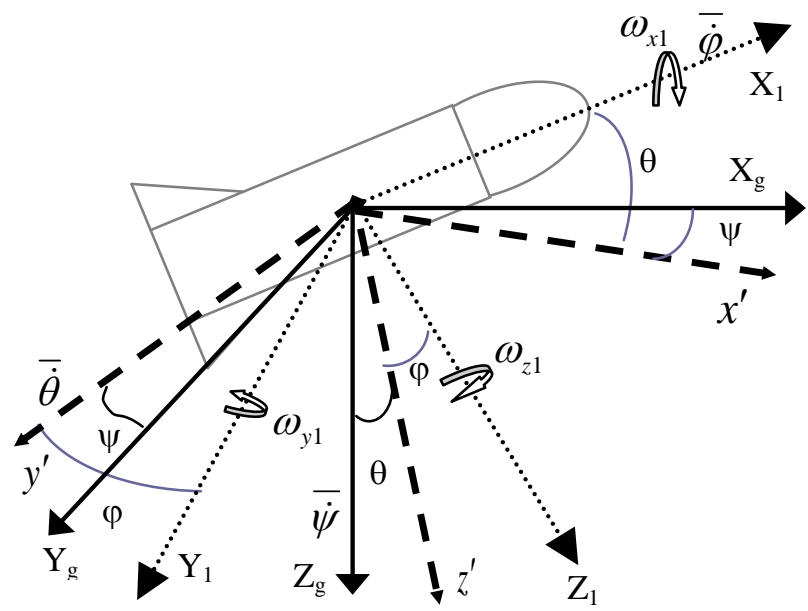

Fig. 1: Ground and Body Axes

$$
\left[\begin{array}{l}
\mathrm{x}_{\mathrm{g}} \\
\mathrm{y}_{\mathrm{g}} \\
\mathrm{z}_{\mathrm{g}}
\end{array}\right]=\mathrm{T}_{\mathrm{bg}}\left[\begin{array}{l}
\mathrm{x}_{1} \\
\mathrm{y}_{1} \\
\mathrm{z}_{1}
\end{array}\right]=\left[\begin{array}{ccc}
\mathrm{c} \psi \mathrm{c} \theta & -\mathrm{s} \psi \mathrm{c} \phi+\mathrm{c} \psi \mathrm{s} \theta \mathrm{s} \phi & \mathrm{s} \psi \mathrm{s} \phi+\mathrm{c} \psi \mathrm{s} \theta \mathrm{c} \phi \\
\mathrm{s} \psi \mathrm{c} \theta & \mathrm{c} \psi \mathrm{c} \phi+\mathrm{s} \psi \mathrm{s} \theta \mathrm{s} \phi & -\mathrm{s} \phi \mathrm{c} \psi+\mathrm{s} \psi \mathrm{s} \theta \mathrm{c} \phi \\
-\mathrm{s} \theta & \mathrm{s} \phi \mathrm{c} \theta & \mathrm{c} \theta \mathrm{c} \phi
\end{array}\right]\left[\begin{array}{l}
\mathrm{x}_{1} \\
\mathrm{y}_{1} \\
\mathrm{z}_{1}
\end{array}\right]
$$

where $\mathrm{X}_{1}, \mathrm{Y}_{1}$, and $\mathrm{Z}_{1}\left(\mathrm{X}_{\mathrm{g}}, \mathrm{Y}_{\mathrm{g}}\right.$, and $\left.\mathrm{Z}_{\mathrm{g}}\right)$ are the vectors components along the board (ground) reference axes. The relative attitude between ground and velocity coordinates is represented by the following transformation matrix: 
$\left[\begin{array}{l}x_{g} \\ y_{g} \\ z_{g}\end{array}\right]=T_{v g}\left[\begin{array}{l}x \\ y \\ z\end{array}\right]=\left[\begin{array}{ccc}c \gamma c \vartheta & c \gamma s \vartheta s \chi-s \gamma c \chi & s \gamma s \chi+c \gamma s \vartheta c \chi \\ s \gamma c \vartheta & c \gamma c \chi+s \gamma s \vartheta s \chi & s \gamma s \vartheta c \chi-s \chi c \gamma \\ -s \vartheta & s \chi c \vartheta & c \vartheta c \chi\end{array}\right]\left[\begin{array}{l}x \\ y \\ z\end{array}\right]$

where $\mathrm{X}, \mathrm{Y}$, and $\mathrm{Z}\left(\mathrm{X}_{\mathrm{g}}, \mathrm{Y}_{\mathrm{g}}\right.$, and $\left.\mathrm{Z}_{\mathrm{g}}\right)$ are the vectors' components along the velocity (ground) reference-axes. The coordinates' transformation from velocity into body coordinates axes can be carried using the following matrix:

$\left[\begin{array}{l}x \\ y \\ z\end{array}\right]=T_{v g}\left[\begin{array}{l}x_{1} \\ y_{1} \\ z_{1}\end{array}\right]=\left[\begin{array}{ccc}c \beta c \alpha & -s \beta & c \beta s \alpha \\ s \beta c \alpha & c \beta & s \beta s \alpha \\ -s \alpha & 0 & c \alpha\end{array}\right]\left[\begin{array}{l}x_{1} \\ y_{1} \\ z_{1}\end{array}\right]$

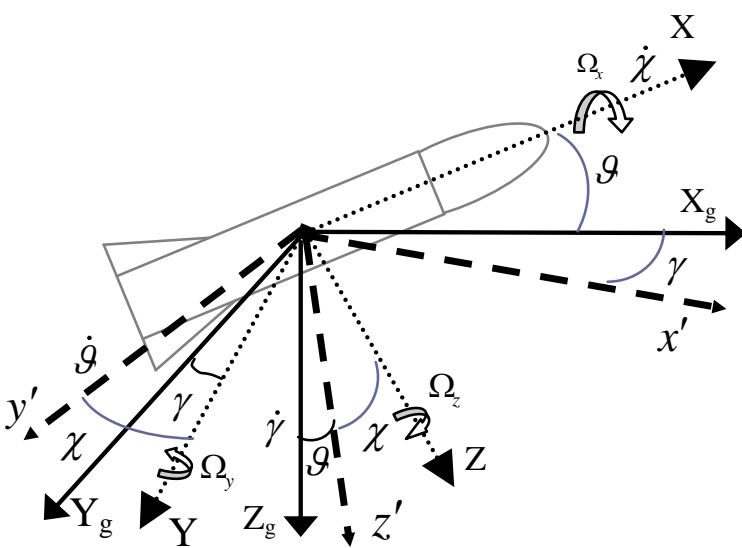

Fig. 2: Ground and Velocity Axes

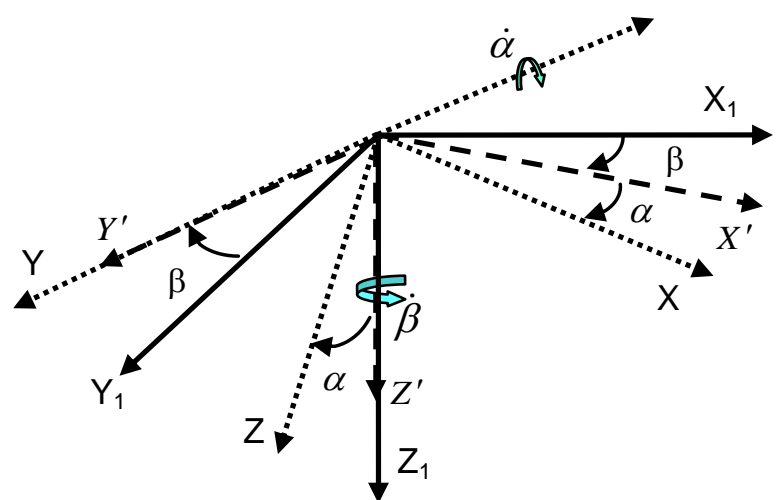

Fig. 3: Velocity and Body axes

\subsection{Acting Forces}

The thrust forces that act on the missile are inclined by angles $\delta_{\mathrm{jp}}$ and $\delta_{\mathrm{jy}}$ in the pitch and yaw planes $[3,6,11]$ as shown in Fig. 4 and it can be resolved as:

$$
\begin{aligned}
& F_{T X_{1}}=F_{T 1} \cos \delta_{j p} \cos \delta_{j y} \\
& F_{T Y_{1}}=-F_{T 1} \cos \delta_{j p} \sin \delta_{j y} \\
& F_{T Z_{1}}=-F_{T 1} \sin \delta_{j p}
\end{aligned}
$$

The thrust jetivator angles $\delta_{\mathrm{jp}}$ and $\delta_{\mathrm{jy}}$ are small enough such that $\mathrm{Eq}^{\underline{\mathrm{n}}}(4)$ can be simplified as:

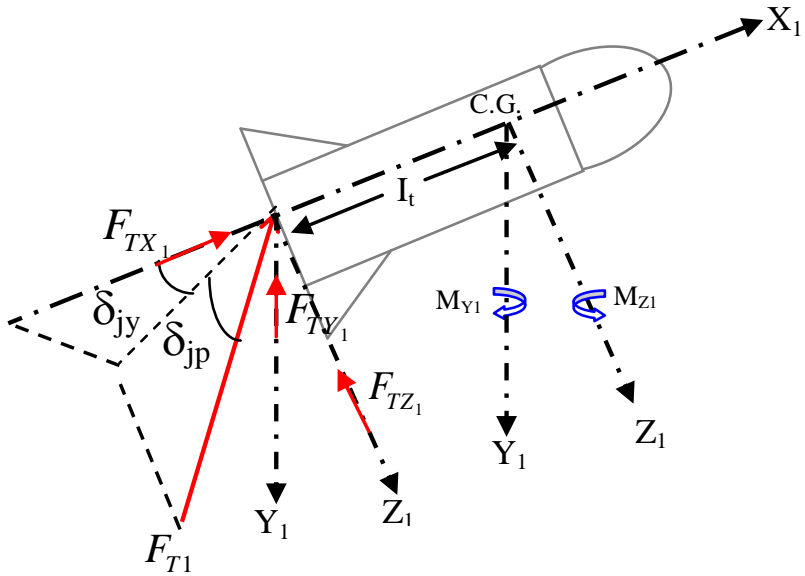

Fig. 4: Thrust forces and moments 


$$
\begin{aligned}
& F_{T X_{1}}=F_{T 1} \\
& F_{T Y_{1}}=-F_{T 1} \delta_{j y} \\
& F_{T Z_{1}}=-F_{T 1} \delta_{j p}
\end{aligned}
$$

Aerodynamic forces have the velocity coordinate system as the mother frame of reference and its components are given $[3,6,11]$ as:

$$
\begin{aligned}
& \mathrm{F}_{\mathrm{AX}}=-\mathrm{C}_{\mathrm{x}} \mathrm{Sq} \\
& \mathrm{F}_{\mathrm{AY}}=-\mathrm{C}_{\mathrm{y}} \mathrm{Sq} \\
& \mathrm{F}_{\mathrm{AZ}}=-\mathrm{C}_{\mathrm{z}} \mathrm{Sq}
\end{aligned}
$$

where $F_{A X}, F_{A Y}$, and $F_{A Z}$ represent, respectively, the drag, lateral, and lift forces along the velocity axes. $S$ represents the characteristic area, (q) represents the dynamic pressure given by $\mathrm{q}=0.5 \rho\left(\mathrm{V}_{\mathrm{M}}\right)^{2}\left[\mathrm{Kg} / \mathrm{m} / \mathrm{sec}^{2}\right], \rho$ is the air density $\left[\mathrm{kg} / \mathrm{m}^{3}\right]$, and $\mathrm{V}_{\mathrm{M}}$ is the missile velocity in $[\mathrm{m} / \mathrm{sec}]$. The aerodynamic coefficients $\mathrm{C}_{\mathrm{x}}, \mathrm{C}_{\mathrm{y}}$, and $\mathrm{C}_{\mathrm{z}}$ are dimension-less and can be written in terms of their derivatives $[3,6,11]$ as:

$$
\begin{aligned}
& C_{x}=C_{x}^{o}+C_{x}^{\alpha^{2}} \alpha^{2}+C_{x}^{\beta^{2}} \beta \\
& C_{y}=C_{y}^{\beta} \beta \\
& C_{z}=C_{z}^{\alpha} \alpha
\end{aligned}
$$

Due to the symmetry of the missile airframe in the pitch and yaw planes, $c_{y}^{\circ}=c_{z}^{\circ}=0$. The missile weight is determined by the relationship $\overline{\mathrm{G}}=\mathrm{m}_{\mathrm{s}} \overline{\mathrm{g}}$, where $\mathrm{m}_{\mathrm{s}}$ is the instantaneous total missile mass and $\bar{g}$ is the vector of gravity acceleration. In some applications, the gravity can be considered constant while the missile mass is varying with time according to the relationship [6]: $m_{s}=m_{o}-\int_{0}^{t} m_{\mathrm{sec}}(t) d t$; where; $\mathrm{m}_{\mathrm{o}}$ is the initial missile mass and $\mathrm{m}_{\mathrm{sec}}$ is the burnt quantity of fuel or propellant per second.

\subsection{Moments Acting on the Missile}

There are three types of moments; thrust moments, the aerodynamic moments and disturbance moments. Thrust moments arise at the time when the thrust does not pass through the missile C.G. and/or does not coincide with the missile longitudinal axis. Thus, it can be resolved into components as shown in Fig. 4 and is given by:

$$
\begin{aligned}
& M_{T X_{1}}=F_{T X_{1}} l_{T X}=F_{T X_{1}} \sqrt{l_{T Y}^{2}+l_{T Z}^{2}} \\
& M_{T Y_{1}}=F_{T Z_{1}} l_{T} \\
& M_{T Z_{1}}=-F_{T Y_{1}} l_{T}
\end{aligned}
$$

where $l_{T}$ is the perpendicular distance between the missile C.G. and the point of lateral thrust forces action, and $l_{T X}$ is the perpendicular distance between longitudinal axis and thrust force line.

The aerodynamic moments originates owing to the fact that the resulting aerodynamic forces act at the missile c.p. and do not pass through the missile c.g. It can be given by its components along the board coordinate system axes [3,6,11] as:

$$
\begin{aligned}
& \mathrm{M}_{\mathrm{AX}_{1}}=\mathrm{m}_{\mathrm{x}_{1}}{\mathrm{Sq} \mathrm{I}_{\mathrm{x}}}_{\mathrm{M}_{\mathrm{AY}_{1}}}=\mathrm{m}_{\mathrm{y}_{1}}{\mathrm{~S} \mathrm{q} \mathrm{l}_{\mathrm{y}}}_{\mathrm{M}_{\mathrm{AZ}_{1}}}=\mathrm{m}_{\mathrm{z}_{1}}{\mathrm{~S} \mathrm{q} \mathrm{l}_{\mathrm{z}}}
\end{aligned}
$$


where: $l_{x}, l_{y}, l_{z}$ are the Characteristic linear dimensions of missile, $\mathrm{S}$ is the characteristic area of missile, and $m_{x_{1}}, m_{y_{1}}$, and $m_{z_{1}}$ are dimensionless aerodynamic coefficients. These functions are usually allocated in the form of graphs obtained by experiments in a wind tunnel. For X-form missile, the aerodynamic moments derivatives are given by $[3,6]$ :

$$
\begin{aligned}
& m_{x_{1}}=m_{x_{1}}^{\omega_{x_{1}}} \omega_{x_{1}} \\
& m_{y_{1}}=m_{y_{1}}^{\omega_{y_{1}}} \omega_{y_{1}}+m_{y_{1}}^{\alpha} \alpha \\
& m_{z_{1}}=m_{z_{1}}^{\omega_{z_{1}}} \omega_{z_{1}}+m_{z_{1}}^{\beta} \beta
\end{aligned}
$$

where $\omega_{x_{1}}, \omega_{y_{1}}$ and $\omega_{z_{1}}$ are the airframe-turn rates along board coordinate axes as shown in Fig. 3.

\subsection{Equations of Motion}

\subsubsection{Force Equations}

The motion of missile is determined by the vector equation $[6,11]$ :

$$
\sum \overline{\mathrm{F}}=\mathrm{m} \overline{\mathrm{J}}=\mathrm{m} \frac{\mathrm{d}\left(\overline{\mathrm{V}_{\mathrm{M}}}\right)}{\mathrm{dt}}=\mathrm{m} \dot{\mathrm{V}}_{\mathrm{M}} \hat{\mathrm{V}}_{\mathrm{M}}+\mathrm{m}\left(\bar{\Omega} \otimes \overline{\mathrm{V}_{\mathrm{M}}}\right)
$$

where $\mathrm{m}$ is the missile mass, $\bar{J}$ is the acceleration of missile, and $\bar{F}$ is the external force acting on the missile, $\Omega$ is the angular velocity of VCS with respect to GCS whose components in VCS are $\Omega_{x}, \Omega_{y}$ and $\Omega_{z}$ as shown in Fig 2. Since $\bar{v}_{M}$ lies along $x$-axis, the velocity components along the $\mathrm{y}$ and $\mathrm{z}$ axis are zero. Consequently, the forward acceleration is $J_{x}=\dot{v}_{M}$ and the normal components are obtained from the cross products; $\bar{\Omega} \otimes \bar{v}_{M}=v_{M} \Omega_{z} \hat{y}-v_{M} \Omega_{y} \hat{z}$. Therefore, the components magnitude of the acceleration vector of the missile c.g. along the VCS axes are obtained as follows:

$$
\bar{J}=\dot{v}_{M} \hat{x}+v_{M} \Omega_{z} y-v_{M} \Omega_{y} \hat{z}
$$

This equation clarifies that the component of angular velocity along the $\mathrm{x}$-axis, aligned with the direction of missile velocity, has no influence upon the acceleration of missile c.g. Now the components of missile acceleration along the axes of the velocity coordinate system are individually written as:

$$
\begin{aligned}
& J_{x}=\dot{v}_{M} \\
& J_{y}=v_{M} \Omega_{z} \\
& J_{z}=-v_{M} \Omega_{y}
\end{aligned}
$$

Substituting $\mathrm{Eq}^{\mathrm{n}}$ (13) into (11) and considering $(3,5,6)$ yield

$$
\begin{aligned}
& \sum F_{x}=m g_{x}+F_{T x}+F_{A x}=m\left(\dot{v}_{x}\right)=m \dot{v}_{M} \\
& \sum F_{y}=m g_{y}+F_{T y}+F_{A y}=m\left(v_{x} \Omega_{z}\right)=m v_{M} \Omega_{z} \\
& \sum F_{z}=m g_{z}+F_{T z}+F_{A Z}=-m v_{M} \Omega_{y}
\end{aligned}
$$

Thus, the acceleration components $J_{x}, J_{y}, J_{z}$ in the velocity reference frame are given by

$$
\begin{aligned}
& J_{x}=g_{x}+\left(F_{T x_{1}} c \beta c \alpha-F_{T y_{1}} s \beta+F_{T z_{1}} c \beta s \alpha-C_{x} s q\right) / m \\
& J_{y}=g_{y}+\left(F_{T x_{1}} s \beta c \alpha+F_{T y_{1}} c \beta+F_{T z_{1}} s \beta s \alpha-C_{y} s q\right) / m \\
& J_{z}=g_{z}+\left(-F_{T x_{1}} s \alpha+F_{T z_{1}} c \alpha-C_{z} s q\right) / m
\end{aligned}
$$


The thrust force components projected into the velocity coordinate system has the form $F_{T}=T_{b v} F_{T_{1}}$, whose components are using the transformation matrix $\mathrm{Eq}^{\mathrm{n}}(3)$ as

$$
\begin{aligned}
& F_{T x}=F_{T x_{1}} c \beta c \alpha-F_{T y_{1}} s \beta+F_{T z_{1}} c \beta s \alpha \\
& F_{T y}=F_{T x_{1}} s \beta c \alpha+F_{T y_{1}} c \beta+F_{T z_{1}} s \beta s \alpha \\
& F_{T z}=-F_{T x_{1}} s \alpha+F_{T z_{1}} c \alpha
\end{aligned}
$$

The acceleration components $J_{x_{1}}, J_{y_{1}}, J_{z_{1}}$ in the body reference frame are given by $\mathrm{J}_{1}=\mathrm{T}_{\mathrm{vb}} \mathrm{J}$, whose components are using the transformation matrix $\mathrm{Eq}^{\mathrm{n}}(3)$ as

$$
\begin{aligned}
& \mathrm{J}_{\mathrm{x}_{1}}=\mathrm{J}_{\mathrm{x}} \mathrm{c} \beta \mathrm{c} \alpha+\mathrm{J}_{\mathrm{y}} \mathrm{s} \beta \mathrm{c} \alpha-\mathrm{J}_{\mathrm{z}} \mathrm{s} \alpha \\
& \mathrm{J}_{\mathrm{y}_{1}}=-\mathrm{J}_{\mathrm{x}} \mathrm{s} \beta+\mathrm{J}_{\mathrm{y}} \mathrm{c} \beta \\
& \mathrm{J}_{\mathrm{z}_{1}}=\mathrm{J}_{\mathrm{x}} \mathrm{c} \beta \mathrm{s} \alpha+\mathrm{J}_{\mathrm{y}} \mathrm{s} \beta \mathrm{s} \alpha+\mathrm{J}_{\mathrm{z}} \mathrm{c} \alpha
\end{aligned}
$$

The position of the velocity coordinate system with respect to the reference ground coordinate system is determined by means of three angles $(\gamma, \vartheta, \chi)$. Therefore, the angular velocity vector $(\Omega)$ of missile rotation with respect to GCS (earth reference system) is given by its components in the direction of VCS axes as $\Omega_{x}, \Omega_{y}$ and $\Omega_{z}$ as shown in Fig. 2. These components can be related to angular rates of Euler's angles $(\dot{\gamma}, \dot{\vartheta}, \dot{\chi})$ through the direction cosines [10] and the algebraic manipulation of its elements yields the angular raters of Euler's angles as follows:

$$
\begin{aligned}
& \dot{\vartheta}=\Omega_{y} \cos \chi-\Omega_{z} \sin \chi \\
& \dot{\gamma} \cos \vartheta=\Omega_{y} \sin \chi+\Omega_{z} \cos \chi \\
& \dot{\chi}=\Omega_{x}+\tan \vartheta\left(\Omega_{z} \cos \chi+\Omega_{y} \sin \chi\right)
\end{aligned}
$$

The components of gravity along the VCS axes are obtained using the transformation matrix $\mathrm{Eq}^{\mathrm{n}}(2)$ as follows:

$$
\left[\begin{array}{l}
g_{x} \\
g_{y} \\
g_{z}
\end{array}\right]=\left[\begin{array}{ccc}
c \gamma c \vartheta & s \gamma c \vartheta & -s \vartheta \\
c \gamma s \vartheta_{s}-s \gamma c \chi & c \gamma c \chi+s \gamma s \vartheta_{s} \chi & c \vartheta_{s} \chi \\
c \gamma s \vartheta_{c} \chi+s \gamma \delta & s \gamma s \vartheta c \chi-c \gamma s \chi & c \vartheta_{c} \chi
\end{array}\right]\left[\begin{array}{l}
0 \\
0 \\
g
\end{array}\right]=\left[\begin{array}{c}
-g s \vartheta \\
g c \vartheta_{s} \chi \\
g c \vartheta_{c} \chi
\end{array}\right]
$$

From the above discussions, it is clear that the equation describing dynamics of guided missile c.g. motion can be summarized as:

$$
\begin{array}{ll}
\dot{v}_{M}=g_{x}+\Phi_{x}-\frac{C_{x} s q}{m} & \\
\dot{\vartheta}=N \cos \chi-K \sin \chi-\frac{g \cos \vartheta}{v_{M}} & \\
\dot{\gamma} \cos \vartheta=N \sin \chi+K \cos \chi & \\
\text { where } & \\
\Phi_{x}=\left(F_{T x_{1}} c \beta c \alpha-F_{T y_{1}} s \beta+F_{T z_{1}} c \beta s \alpha\right) / m & n_{\circ}+n_{\alpha} \alpha \\
n_{\circ}=-\frac{1}{m v_{M}}\left(-F_{T x_{1}} s \alpha+F_{T z_{1}} c \alpha\right) & \\
K=k_{\circ}+k_{\beta} \beta & k_{\circ}=\frac{1}{m v_{M}}\left(C_{z}^{\alpha} s q\right) \\
k_{\beta}=-\frac{1}{m v_{M}}\left(C_{T x_{1}} s \beta c \alpha+F_{T y_{1}} c \beta+F_{T z_{1}} s \beta s \alpha\right)
\end{array}
$$




\subsubsection{Moment Equations}

The external moments acting on a body equal to the time rate of change of its moment of momentum (angular momentum). The time rates of change are all taken with respect to inertial space and can be expressed as follows [6,11]:

$$
\sum M=\left.\frac{d(H)}{d t}\right|_{I}
$$

where the subscript (I) indicates that the time rate of change of the vector is obtained with respect to inertial space. Due to symmetry of missile configuration, the products of inertia are neglected $[6,11]$ and the equations of missile rotation around its c.g. are obtained as follows:

$$
\begin{aligned}
& \dot{\theta}=\omega_{y_{1}} \cos \phi-\omega_{z_{1}} \sin \phi \\
& \dot{\psi}=\left(\omega_{z_{1}} \cos \phi+\omega_{y_{1}} \sin \phi\right) / \cos \theta \\
& \dot{\phi}=\omega_{x_{1}}+\left(\omega_{z_{1}} \cos \phi+\omega_{y_{1}} \sin \phi\right) \tan \theta \\
& M_{x_{1}}=I_{x x} \dot{\omega}_{x_{1}}+\left(I_{z z}-I_{y y}\right) \omega_{z_{1}} \omega_{y_{1}} \\
& M_{y_{1}}=I_{y y} \dot{\omega}_{y_{1}}+\left(I_{x x}-I_{z z}\right) \omega_{z_{1}} \omega_{x_{1}} \\
& M_{z_{1}}=I_{z z} \dot{\omega}_{z_{1}}+\left(I_{y y}-I_{x x}\right) \omega_{x_{1}} \omega_{y_{1}}
\end{aligned}
$$

where $\mathrm{I}_{\mathrm{XX}}, \mathrm{I}_{\mathrm{YY}}$, and $\mathrm{I}_{\mathrm{ZZ}}$ are moments of inertia components along the board coordinate axes. The manipulation of $\mathrm{Eq}^{\mathrm{n}}$ (20b) yields the dynamic equations of missile rotation around its c.g. as follows $[9,11,13]$ :

$$
\begin{aligned}
& \dot{\omega}_{x_{1}}=M_{x_{1}} / I_{x x}+\left(I_{y y}-I_{z z}\right) \omega_{y_{1}} \omega_{z_{1}} / I_{x x} \\
& \dot{\omega}_{y_{1}}=M_{y_{1}} / I_{y y}+\left(I_{z z}-I_{x x}\right) \omega_{x_{1}} \omega_{z_{1}} / I_{y y} \\
& \dot{\omega}_{z_{1}}=M_{z_{1}} / I_{z z}+\left(I_{x x}-I_{y y}\right) \omega_{y_{1}} \omega_{x_{1}} / I_{z z}
\end{aligned}
$$

\subsubsection{Geometrical Relations}

The relative attitude between reference frames can be described by considering the Euler's angles $(\gamma, \vartheta, \chi)$ of VCS with respect to GCS, $(\alpha, \beta)$ angles of VCS with respect to BCS, angles $(\psi, \theta, \varphi)$ between BCS with respect to GCS and combined rotations of reference frames as shown in Fig. 5; i.e.

$$
\begin{aligned}
& x=T_{b v} x_{1} \\
& x_{1}=T_{g b} x_{g} \\
& x=T_{g v} x_{g}
\end{aligned}
$$

Manipulating these equations yields $T_{g b}=T_{v b} T_{g v}$, from which only three elements are selected from the above equations to yield the geometrical relations as follows:

$$
\begin{aligned}
& \theta=\vartheta+\beta \sin \chi+\alpha \cos \chi \\
& \phi=\psi+\alpha \tan \vartheta \sin \chi-\beta \tan \vartheta \cos \chi \\
& \psi=\gamma+((\alpha \sin \chi-\beta \cos \chi) / \cos \vartheta)
\end{aligned}
$$




\section{6DOF Flight Simulation Model}

A flight simulation model is designed in a modular structure to obtain the flight trajectory of the missile with programs built within $\mathrm{C}++$ and MATLAB environments. The block diagram that shows the flow of data among the various modules is given in Fig. 6a. The simulation model can be broken down into the following major parts: missile-target geometry, guidance, control, thrust, force-moments, aerodynamics, and missile [4,5]. As shown in Fig. $6 a$ the module denoted by CRB1 represents the transformation between the reference and the board coordinate systems, which is given by $\mathrm{Eq}^{\underline{\underline{n}}}$ (1). The output velocity corrupted with the wind is applied to the aerodynamic module, which calculates the aerodynamic forces and moments. The thrust module computes the thrust force components along

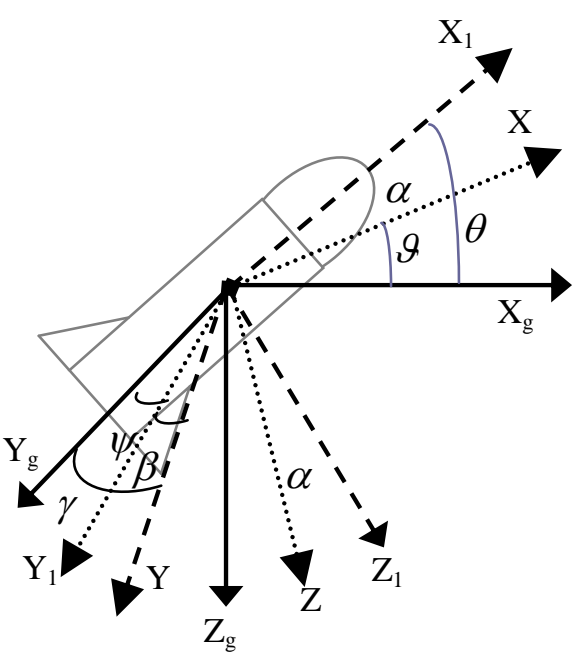

Fig. 5: Geometrical relation the missile axes according to the jetivator deflection angles $\delta_{\mathrm{jp}}$ and $\delta_{\mathrm{jy}}$ in pitch and yaw planes, respectively. The deflection angles are generated in the jet module which stimulated by the input voltages generated from the autopilot module. The autopilot calculates these voltages by comparing the guidance commands, which represent the desired missile heading and the actual missile heading. The difference between both is processed in the autopilot electronics to-eventually produce the desired jetivator deflection. The actual missile heading is obtained via two free gyros mounted perpendicular to each other in the missile lateral plane. The solution of the missile dynamical equations of motion is carried out numerically in the vehicle dynamics module where Rung-Kutta 4 method is employed. The outputs of the vehicle (missile) dynamics module are the missile linear and angular acceleration components obtained in the board coordinate system. Through the CBR module, the missile velocity and turn rates expressed in the reference coordinate system are obtained. Integrating these velocities yields the missile instantaneous position. The module denoted by CRB2 represents the transformation between the reference coordinate system and the board coordinate system for gravity. Inside the geometry module the missile instantaneous position is compared with the target position and the relative error is obtained. The guidance module generates guidance commands in addition to the pre-programmed commands which applied to the missile autopilot just after launch. The purpose of these commands is to gather the missile to the target line of sight with no intervention from the guidance commands in the initial flight period. The joystick commands are generated using a tuned PD controller and the overall flowchart describing the system modules is shown in Fig. 6 b.

\subsection{Missile-Target Geometry Module}

It determines the relative position and calculates guidance parameters that are used by the missile guidance generation system to steer the missile in space. A simplified block diagram of this process is shown in Fig. 7, where the target tracker in the system is a manual telescope fixed on the sight station but missile tracker is merely an electric tracker. This function receives data expressed in the reference frames, concerning the new position, and velocity of the missile and target. By using these data $[X, Y, Z]$ for missile and $\left[X_{t}, Y_{t}, Z_{t}\right]$ for target. The function computes missile and target parameters (range, range rate, LOS angles, and LOS angles rate). The missile target parameters are deduced through the following relations: 


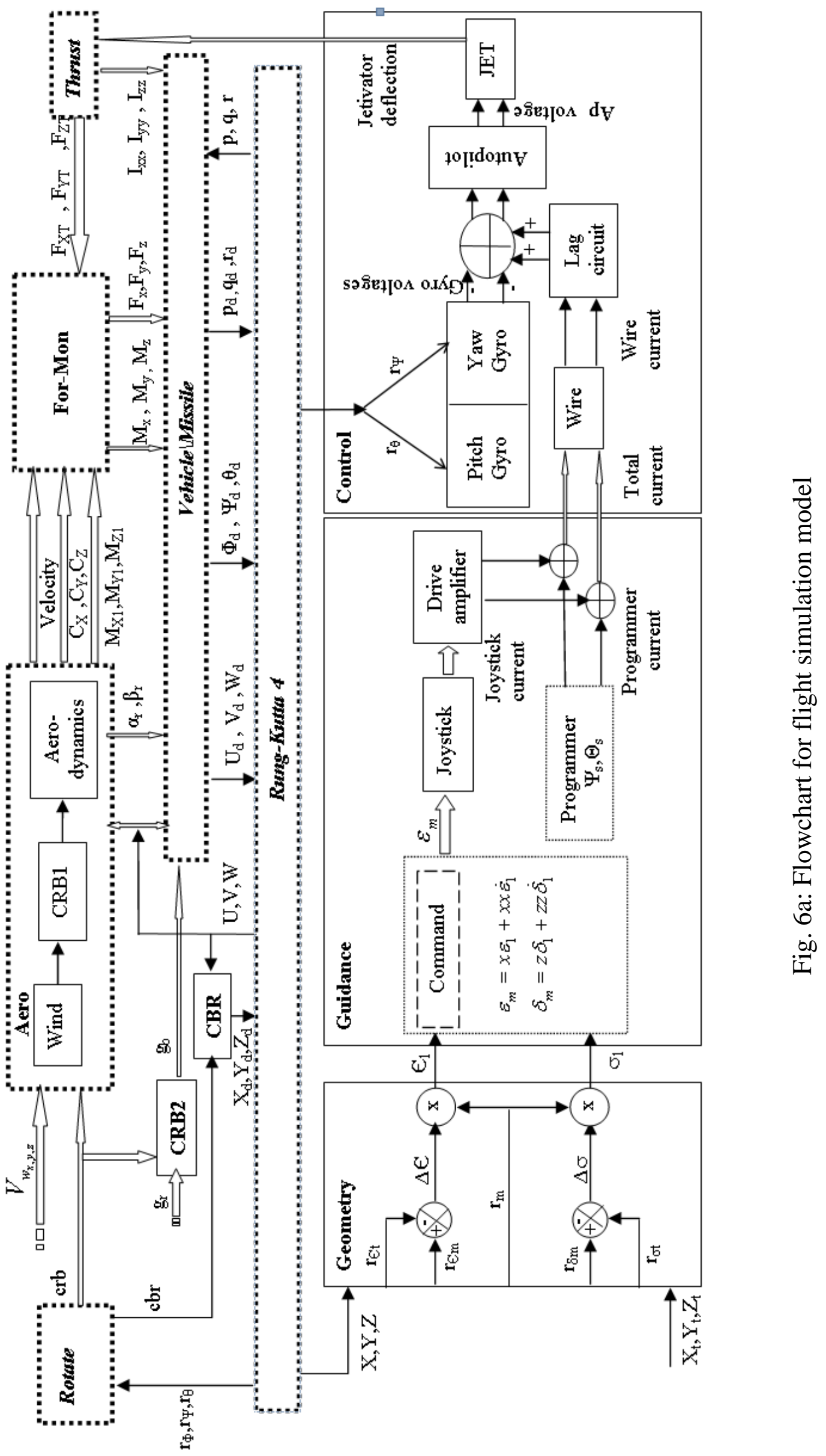




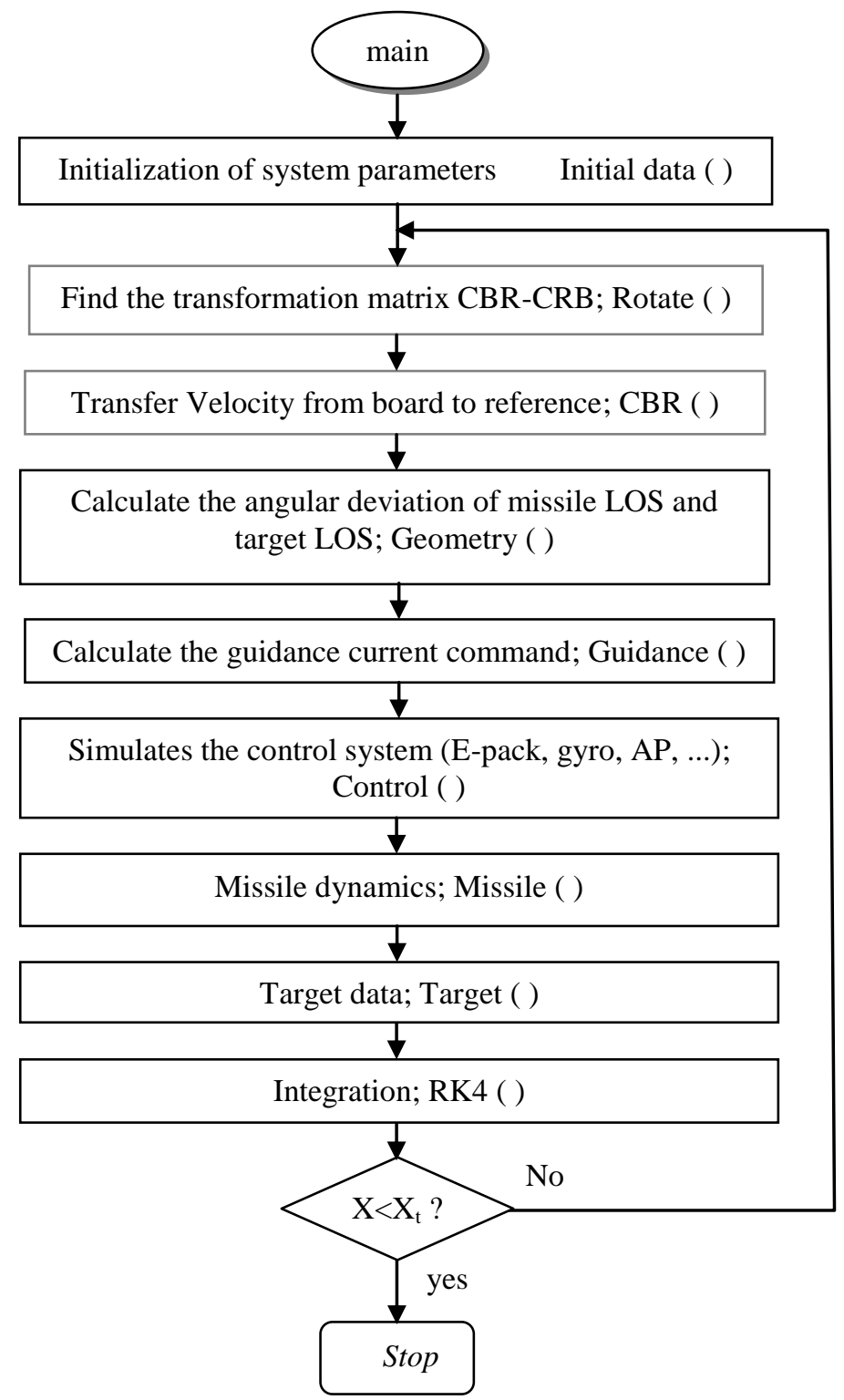

Fig. 6b: Flowchart of the 6DOF flight simulation model

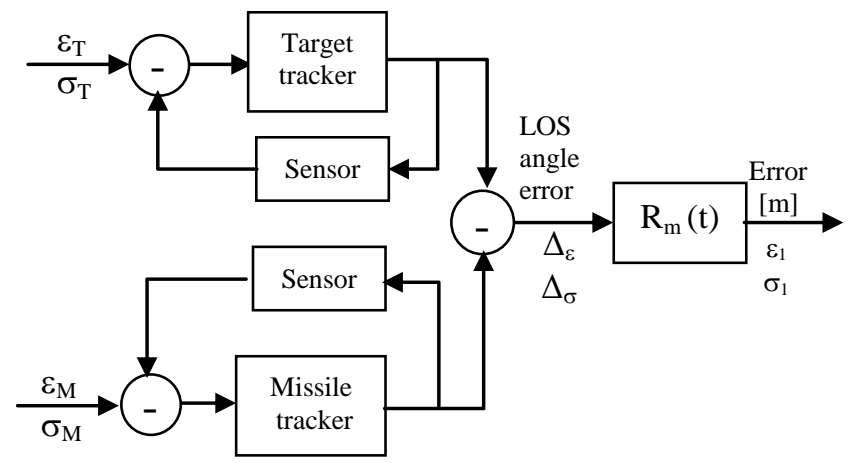

Fig. 7: Simplified BD of Missile-Target Geometry 


$$
\begin{aligned}
& \varepsilon_{m}\left(\varepsilon_{t}\right)=\tan ^{-1}\left(-\frac{Z\left(Z_{t}\right)}{X\left(X_{t}\right.}\right) \\
& \sigma_{m}\left(\sigma_{t}\right)=\tan ^{-1}\left(-\frac{Y\left(Y_{t}\right)}{X\left(X_{t}\right.}\right) \\
& r_{m}=\sqrt{X^{2}+Y^{2}+Z^{2}} \\
& r_{t}=\sqrt{X_{t}^{2}+Y_{t}^{2}+Z_{t}^{2}}
\end{aligned}
$$

where $\varepsilon_{M}\left(\varepsilon_{T}\right)$ is the elevation LOS angle, $\sigma_{M}\left(\sigma_{T}\right)$ is the azimuth LOS angle, and $r_{m}\left(r_{t}\right)$ is the range for missile (target).

\subsection{Guidance Module}

This module calculates the total commanded current generated in the wire command link of the missile system, as shown in Fig. 8, where $\varepsilon_{1}, \sigma_{1}$ is the LOS angular errors for the two planes expressed in meters. The command section implements the LOS guidance law and it can be characterized as a dynamic correction function in the closed loop of the guidance process which provides shaping of the commands to control the guided missile. The method of control is based on the estimation of the lateral displacement of the missile from the target LOS.

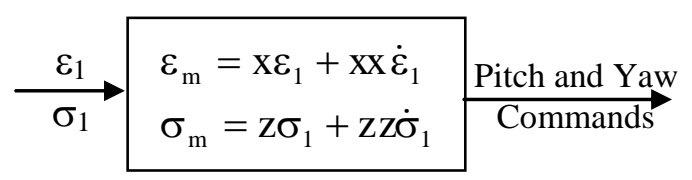

Fig. 8: Command section The linear displacement error is given by:

$$
\begin{aligned}
& \mathrm{h}_{\varepsilon}=\mathrm{r}(\mathrm{t})\left(\varepsilon_{\mathrm{t}}-\varepsilon_{\mathrm{m}}\right) \\
& \mathrm{h}_{\delta}=\mathrm{r}(\mathrm{t})\left(\sigma_{\mathrm{t}}-\sigma_{\mathrm{m}}\right)
\end{aligned}
$$

Using conventional control the commands passed to the missile are proportional to the linear error. The control signals depend not only on the error signals but also on its derivative with the aim of increasing the relative stability and improving the transients in the guidance system. Two signals are summed up at the input of the wire link as shown in Fig. 9 which represents the Programmer and Joystick currents. That is, the total output current is the summation of the programmer current and the joystick current.

The purpose of the guidance system is to guide the missile in pitch- and yaw-planes so that it gathers the missile and flies it along the desired LOS. The yaw and pitch demands are in the form of D.C. levels, computed by the programmer electronic control (PEC) using input information relating to the position of the weapon system and target, Fig. 10. The demands are fed to the missile through a 3-wire link in the missile control wire. These demands are programmed so that, initially they consist of step demand, which cause the missile to fly towards the sightline, and then constant demands which hold the flight path parallel with the sightline [12]. The programmer is a computer consisting, basically, of a yaw channel, a pitch channel and timing and calculation of separation parameters. The yaw and pitch drive amplifiers are identical current generators, the outputs of which are fed to the three-wire link to the control system in the missile. The yaw and pitch demands return form the missile via a common line along which the voltage drop is proportional to the sum of the two demand currents. This voltage drop is high when the demands are of like polarities, i.e. up and right or down and left. 


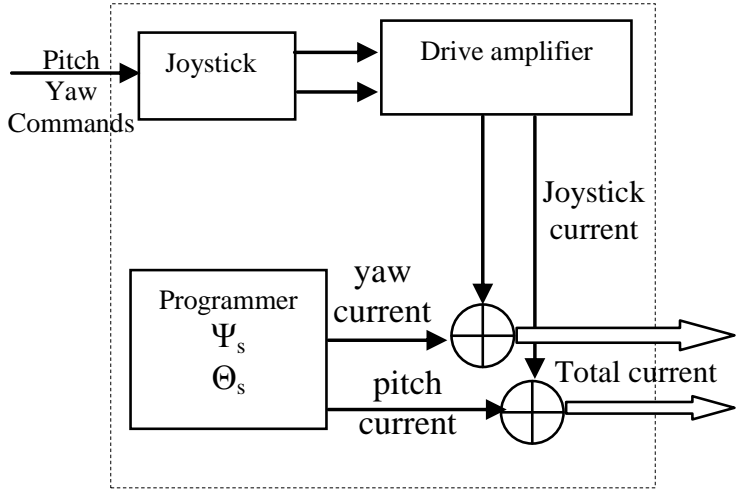

Fig. 9: Guidance section

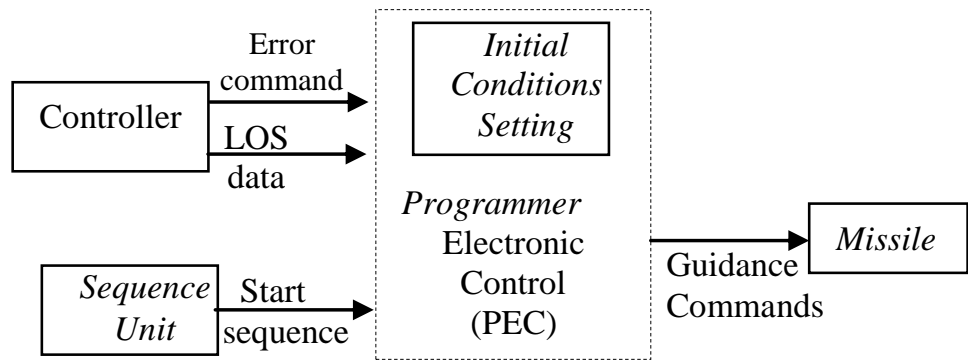

Fig. 10: Programmer function in guidance and control

The pitch command starts at time $(\mathrm{t}=0.0)$ to produce a pitch demand $\theta_{\mathrm{p}}$ so that the missile flies along the sight line at an incidence angle $\left(3.66^{\circ}\right)$ required for aerodynamic lift. Basically, the demand required to guide the missile along the flight path consists of $\left(\theta_{\mathrm{s}}+3.66^{\circ}-35^{\circ}\right)$ and it is applied gradually. The initial value of $\theta_{\mathrm{p}}$ is selected such that the missile reaches a height not less than 3 meters above the launcher, at a distance of 9 meters in front of the launcher. Therefore, the pitch demand consists of an initial step demand during the period of 1.37 seconds to ensure that the missile gathers to the sight line as quickly as possible thereby achieving a good minimum range performance. In addition, it consists of an exponential command which commences after the step demand so that the missile descends and levels out to its correct pitch attitude. Thus, the azimuth programmer equations [11] are:

$$
\begin{aligned}
& \psi_{\mathrm{p}}=\left(\psi_{A}+\psi_{B}\right)\left(1-e^{-t / \tau_{\psi}}\right) \\
& \psi_{A}=\psi_{B} \\
& \psi_{B}=K_{1} \psi_{S}
\end{aligned}
$$

where $K_{1}=0.3$ if time of flight $<T_{y}$ and zero if time of flight $>T_{y}, T_{y}$ is the end of gathering to sight line phase of engagement, and $\psi_{\mathrm{s}}$ is the angle between sight forward axis and LOS in yaw plane as shown in Fig. 11a. Similarly, the elevation programmer equations are:

$$
\begin{aligned}
& \theta_{\mathrm{p}}=-\left(\theta_{\mathrm{A}}-\theta_{\mathrm{B}} \mathrm{e}^{-\mathrm{t} / \tau_{\theta}}\right) \\
& \theta_{\mathrm{A}}=35^{\circ}-\left(\theta_{\mathrm{s}}+3.66^{\circ}\right) \\
& \theta_{\mathrm{B}}=\mathrm{E}_{\circ}+\mathrm{K}_{\mathrm{s}} \theta_{\mathrm{s}}
\end{aligned}
$$

where $\tau_{\theta}=2.5 \mathrm{sec}, \mathrm{K}_{\mathrm{s}}=0.15, \mathrm{E}_{\mathrm{o}}$ is the temperature compensation term ( $13.7^{\circ}$ normally), $\theta_{\mathrm{c}}$ is the crest angle, and $\theta_{\mathrm{s}}$ is the angle between sight forward axis and LOS in pitch plane as shown in Fig. 11b. 


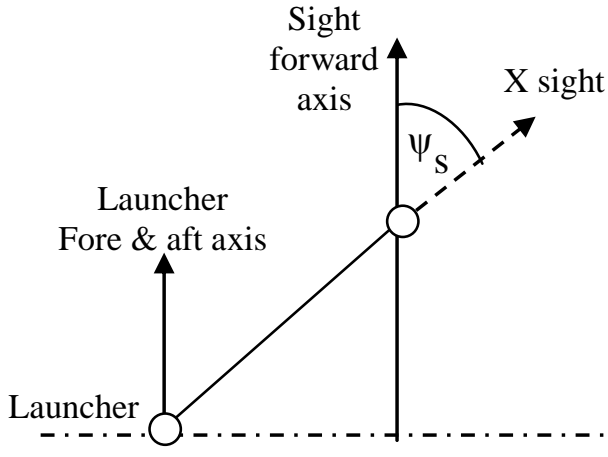

Fig. 11a: Tactical angles in yaw plane

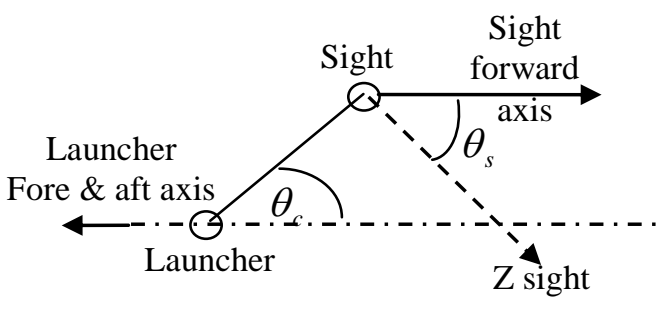

Fig. 11b: Tactical angles in pitch plane

\subsubsection{Joystick Function}

The command generated by the command section of the guidance module is applied to the joystick transfer function to generate joystick voltages which is applied to the derive amplifier which generates drive amplifier currents. The limitation on the total current must be put into consideration in the design methodology, such that the max normalized current generated in pitch plane (up) does not exceeds $(14 \mu \mathrm{A})$ and (down) does not less than $(-29 \mu \mathrm{A})$. While, the max current generated in yaw plane (right) does not exceeds $(29 \mu \mathrm{A})$ and (left) does not less than $(-29 \mu \mathrm{A})$. The programmer and joystick currents generated from reference experiments are shown in Fig. 12 and they would be used in designing necessary controllers.

\subsection{Control Function}

This routine simulates the current of the missile control system, which involves the missile electronic package circuitry and the rate gyro feedback as shown in Fig. 13. This subroutine converts the derived guidance signal, with some feedback signals, into control jetivator deflection commands. The feedback signals through instruments are represented by their transfer functions. The module output is applied to the thrust section to calculate the thrust force of a missile.

\subsection{Thrust Function}

This routine calculates the thrust force of the missile, where the typical parameters are: normalized booster thrust $=180[\mathrm{~N}]$, time of booster $=5-6[\mathrm{sec}]$, sustainer thrust $=48[\mathrm{~N}]$, and time of sustainer $=20[\mathrm{sec}]$. Experimental measurements show that there are uncertainties in the values of thrust in booster and sustainer periods, mainly due to aging. The routine also considers the limitations of jetivator deflection angles given by 10.5 degree in pitch and yaw. Then the thrust forces are calculated as illustrated in $\mathrm{Eq}^{\underline{\underline{n}}}(4)$.

\subsection{Force-Moment Function}

This function calculates all forces and moments acting on the missile, which necessitates other functions such as the aerodynamic function that represents the linearized aerodynamical model for all aerodynamic coefficients and derivatives used in the computation of forces and moments. 


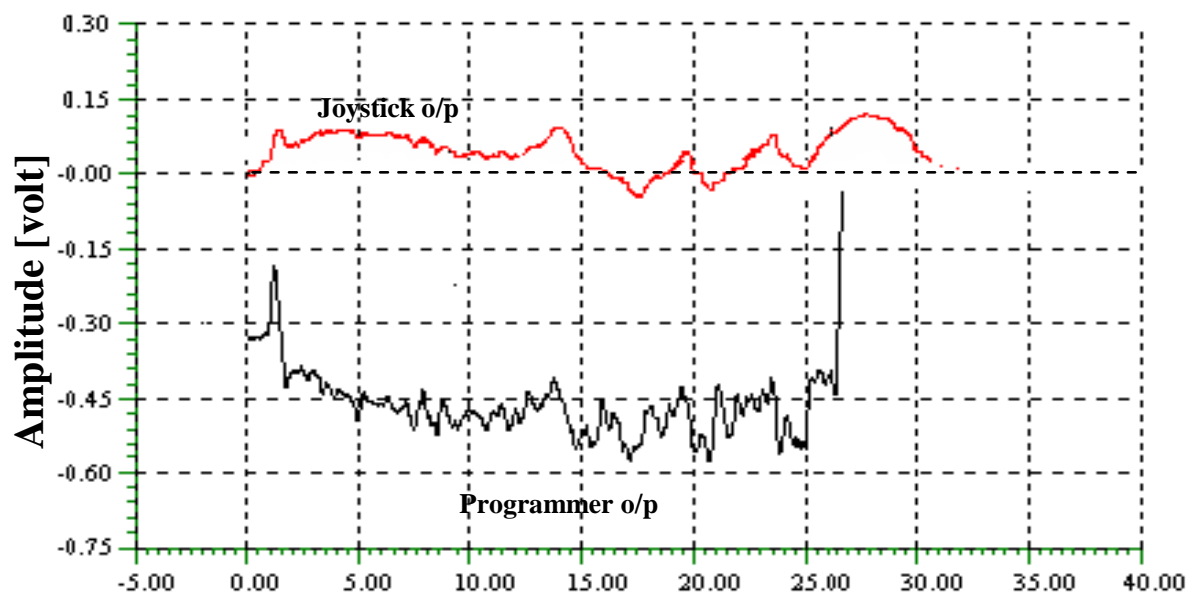

(a) Time $[\mathrm{sec}]$

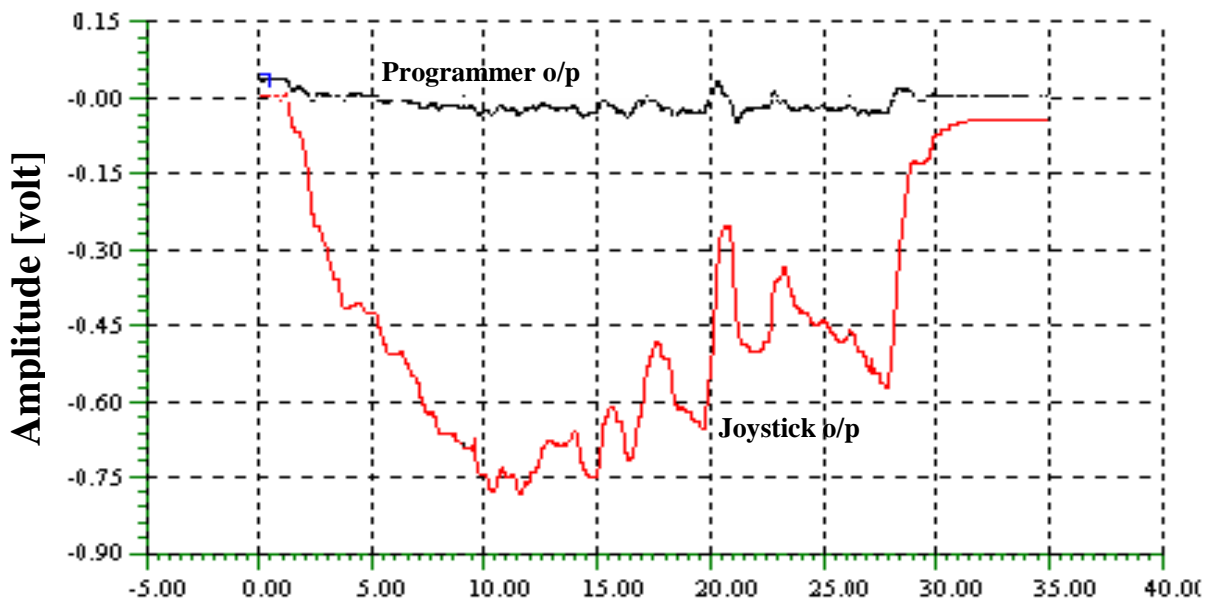

(b) Time [sec]

Fig. 12: Typical guidance volts (currents) for (a) Pitch and (b) yaw channels

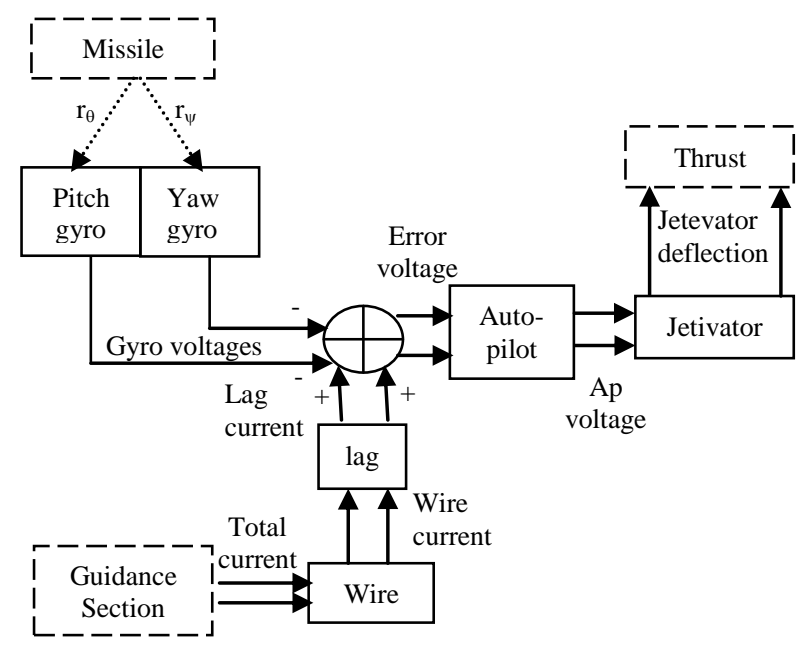

Fig. 13: Control section 


\subsection{Aerodynamic Function}

This routine calculates the resultant missile speed $V_{m}$ which is given by:

$$
v_{M}=\sqrt{u^{2}+v^{2}+w^{2}}
$$

where $\mathrm{U}, \mathrm{V}$, and $\mathrm{W}$ are the velocity components along board coordinate axes. Also the aerodynamic force and moment coefficients are usually defined as functions of angle of attack $(\alpha)$, sideslip angle $(\beta)$, and other flight parameters. In reference to Fig. 3 , the relationships between the velocity components and the firing angles are:

$$
\begin{aligned}
& \alpha=\tan ^{-1}(w / u) \\
& \beta=\tan ^{-1}(v / u)
\end{aligned}
$$

Note that the missile function solves Euler's equations via calling other necessary functions including transformation, gravity, and forces and moments.

\subsection{Auxiliary Modules}

There are some auxiliary functions including Initialize module that is devoted to initialize all the missile and target initial states (position, velocity, and acceleration) with different gains for the guidance and control loops; the Integration module which uses a fourth order rungekutta method to perform the numerical integration for all the states of differential equations describing the model; the Output module where output parameters describing the behavior of the system are stored and used for the system performance analysis; the Rotate module that is used to calculate the transformation matrix from board to reference coordinate system or its transpose; and the Miss-distance module which is devoted to calculate the miss-distance (the closest distance between the target and the missile).

\section{Flight Simulation Analysis}

This section is devoted for evaluating pre-calculated parameters (aerodynamic and dynamic parameters) to get a fair match between the simulated and real flight trajectories. It yields the appropriate and more accurate model that can be used for the guidance and control design, discussed later in the next two paper-parts.

\subsection{Thrust Uncertainties}

One source of uncertainties in the system is the boost thrust values. The velocity is taken as a measuring factor to calculate the uncertainties in the boost thrust values. Fig. 14 shows some experimental velocity profiles recorded from different experimental engagements. Inspection of the experimental flight trajectories reveals that the velocity of the missile varies from run to run and reaches 148-152 [m/sec] at the end of booster and reaches 179-185 [m/sec] at the end of sustainer. That is the variations in velocity profiles are within limits $(-7 \%)$ from the nominal value. This variation in velocity profiles is generated from $(-13 \%)$ uncertainties in the thrust values. The difference between the experimental velocity profiles and the theoretical (nominal) velocity profiles gained from flight simulation model can be minimized by changing the values of thrust in both booster and sustainer periods. The moderate thrust profile is considered with the reference and perturbation data, the sample of simulation results is shown in Fig. 15.

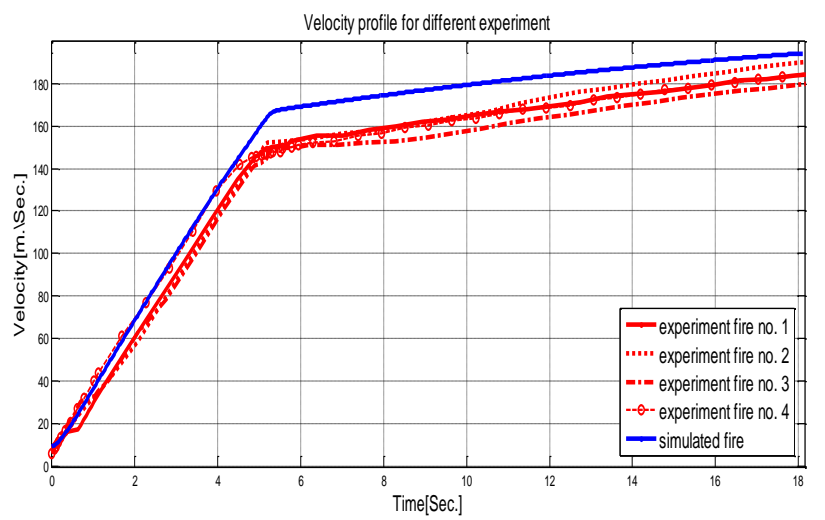

Fig. 14: Experimental velocity profiles for different engagements 


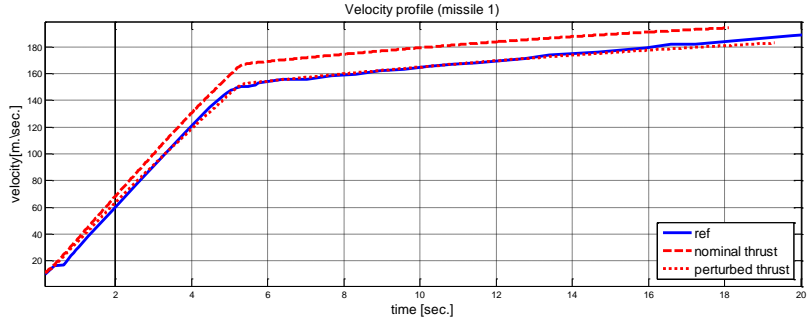

Fig. 15a: Velocity profile (missile no.1)

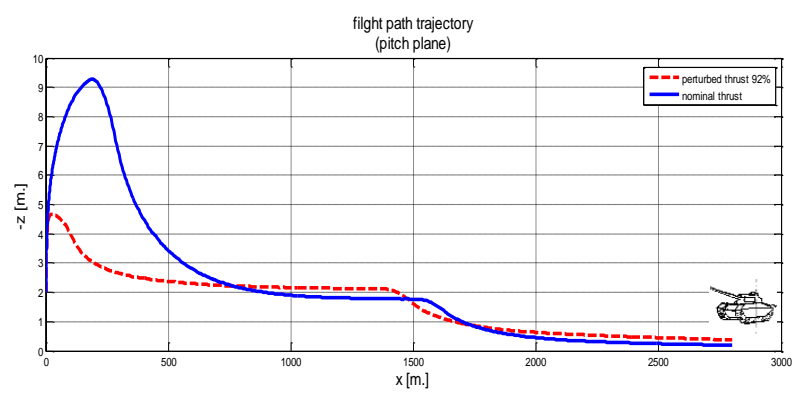

Fig. 15c: Pitch trajectory profile (missile no.1)

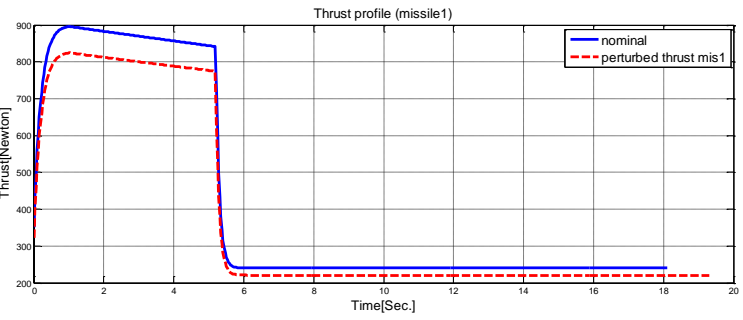

Fig. 15b: Thrust profile (missile no.1)

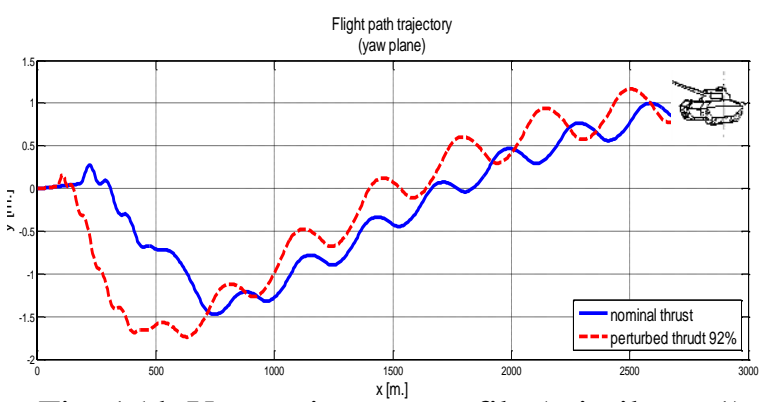

Fig. 15d: Yaw trajectory profile (missile no.1)

The simulation results reveal that the simulated velocity profile can be considered consistent with the real one by adjusting the thrust simulated value. The simulation gives an indication for the uncertainty margin in the boost thrust value for real missile which have a successful engagement for about (92\%) of normal thrust value. It must be put into consideration that the missiles which have ground impact in the early flight period have a thrust value lower than this margin.

\subsection{Aerodynamic Uncertainties}

Another source of uncertainties is attributed to the anomalies in aerodynamic coefficients, for which Fig. 16 and the remainder results show the effect of change in the aerodynamic coefficients in pitch- and yaw-flight path trajectories under different conditions of thrust
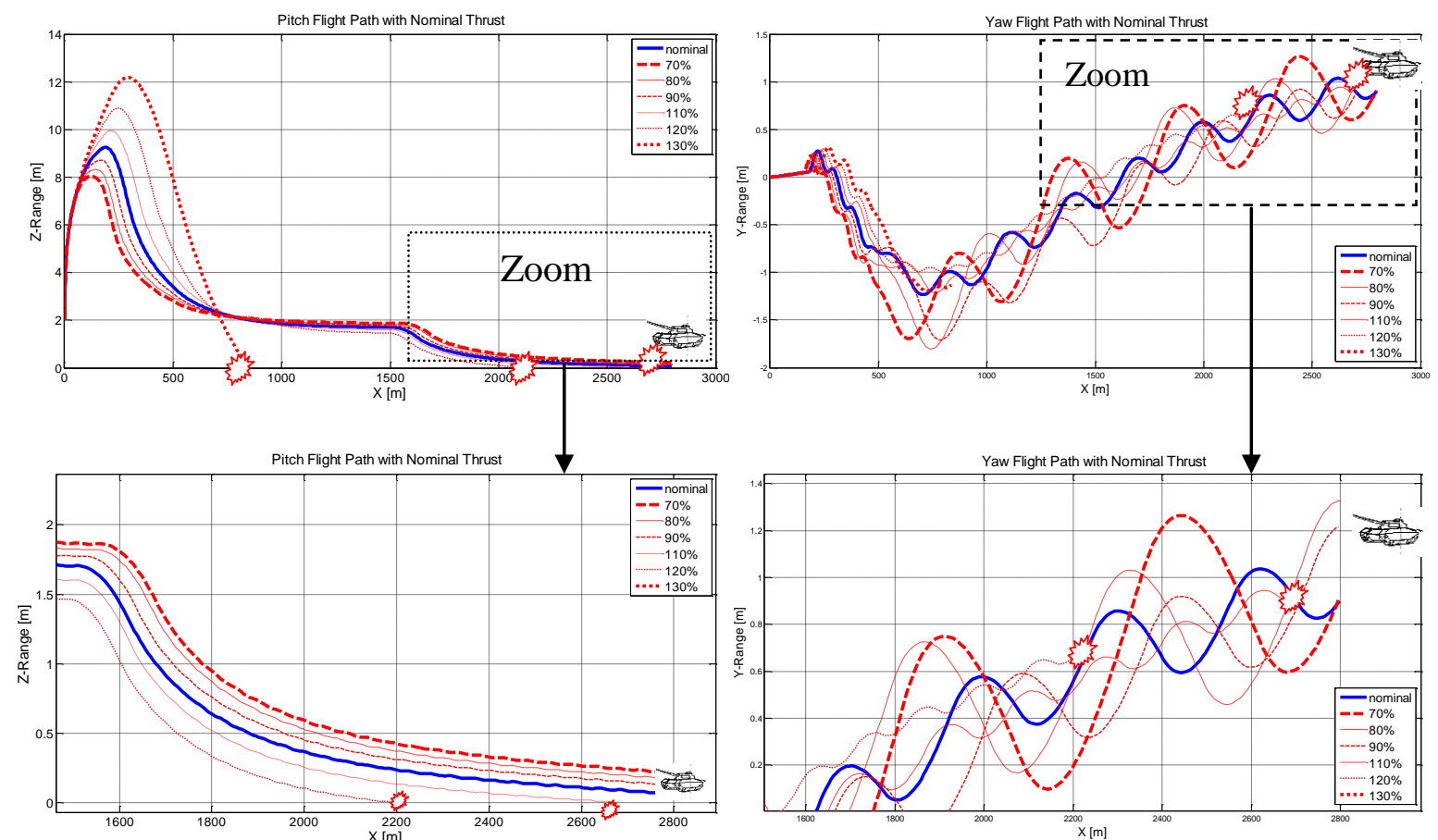

Fig. 16: Pitch Trajectory for different A.D.C. values at nominal thrust 
value. It can be noticed that the difference in miss distance obtained from different cases and the cases where the ground impact occurred. For nominal thrust value the ground impact occurred at $+10 \%$ of aerodynamics coefficient in 2670 meter and at $+20 \%$ in 2190 meter and at $+30 \%$ in 852 meter and for $-14 \%$ thrust value the ground impact occurred at $+10 \%$ of aerodynamics coefficient in 575 meter and at $+20 \%$ in 542 meter and at $+30 \%$ in 2330 meter. In other words the figure reveals that the change in aerodynamic coefficients can be considered as one source of uncertainties in the desired system.

\subsection{Wind Velocity}

The third source of uncertainties is attributed to the effect of wind velocity. Thus, Figs $(17,18,19)$ show the effect of wind velocity on the missile flight path trajectories i.e. wind velocities in $\mathrm{x}, \mathrm{y}$, and $\mathrm{z}$ directions can be considered as a source of uncertainties in this work.

\section{Wind velocity in $\mathrm{X}$-direction}
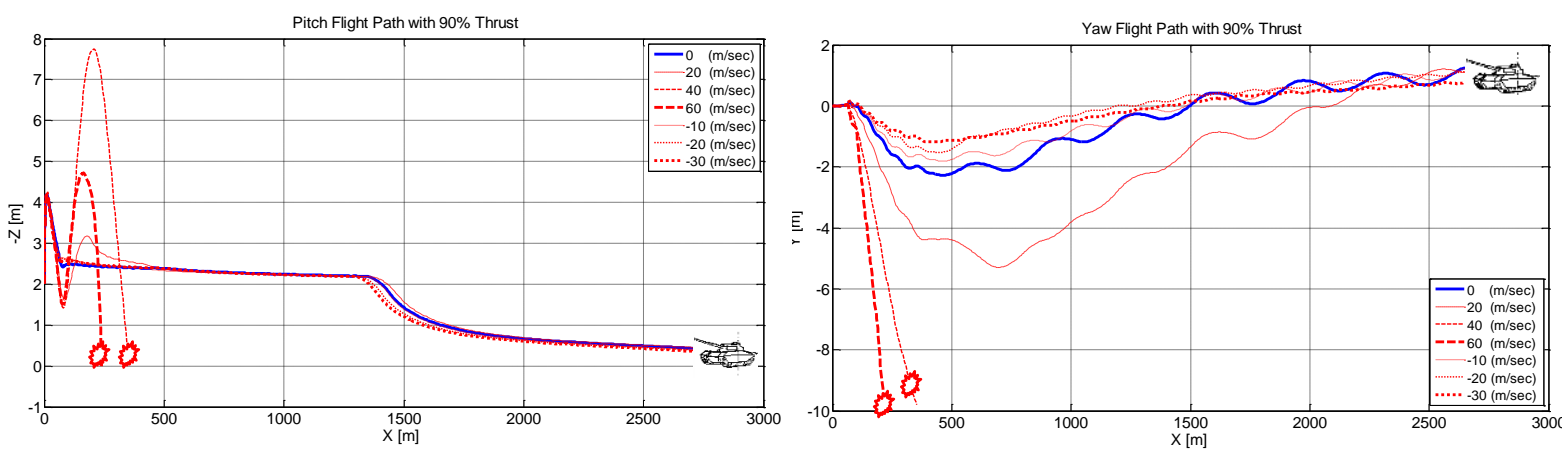

Fig. 17: Pitch and Yaw Trajectories at $90 \%$ thrust for different $\mathrm{V}_{\mathrm{wx}}$

\section{Wind velocity in Y-direction}
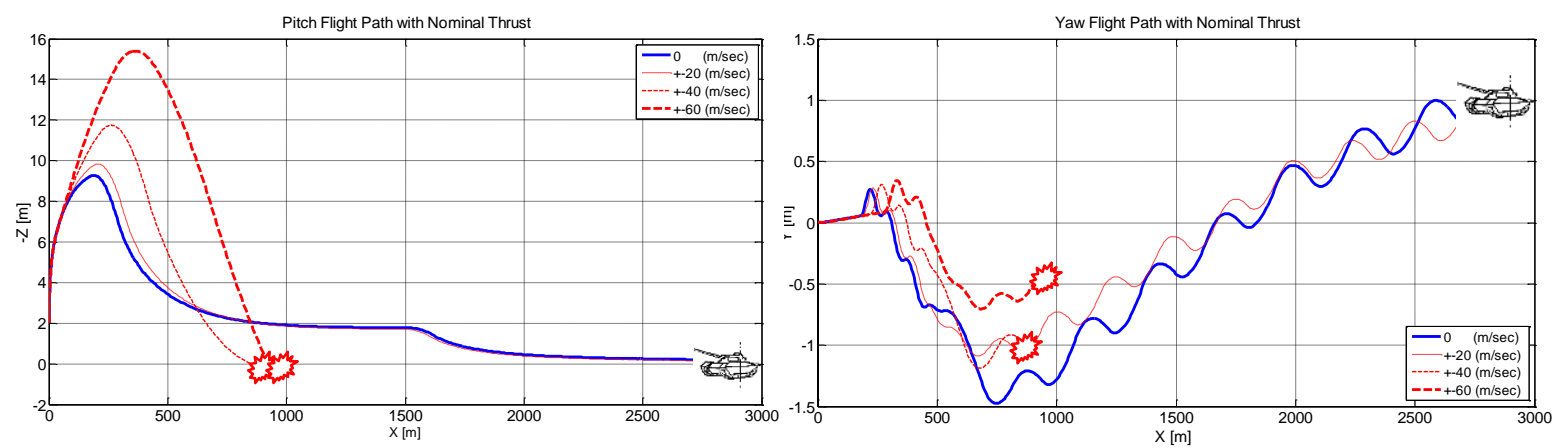

Fig. 18: Pitch and Yaw Trajectories at nominal thrust for different $V_{w y}$

Wind velocity in $\mathrm{Z}$ direction
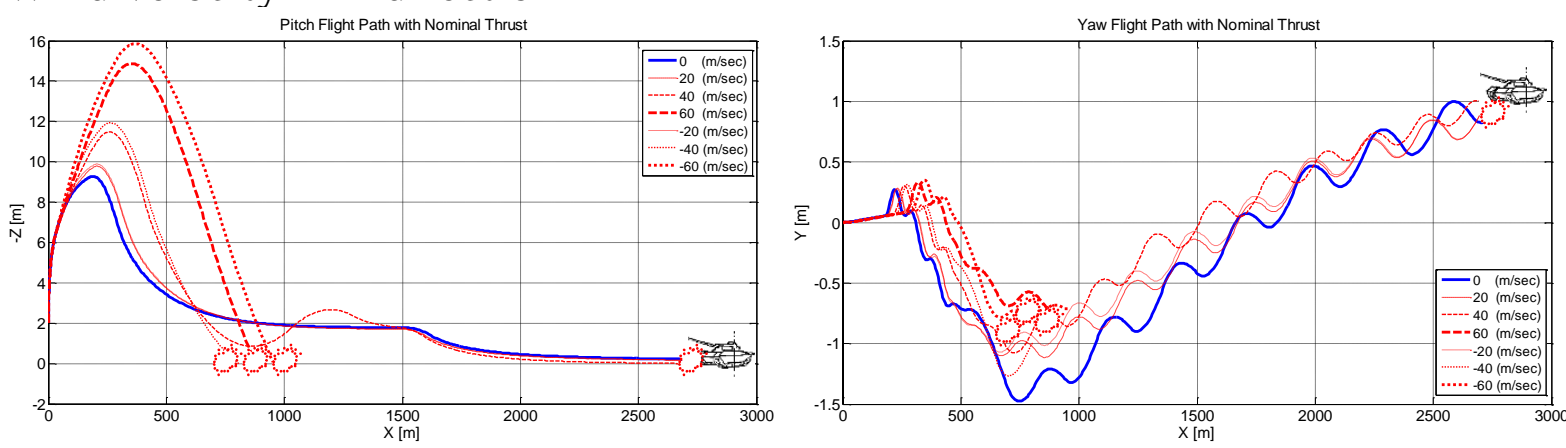

Fig. 19: Pitch and Yaw Trajectories at nominal thrust for different $\mathrm{V}_{\mathrm{wz}}$ 


\subsection{Evaluation of the Flight Simulation Model}

Let us now evaluate the established flight simulation model by comparing its output flight path trajectory with reference trajectories. The simulation is conducted with nominal and perturbed values and depicted with the reference as shown in Fig. 20. This Figure reveals that the established flight simulation which had programmed within both $\mathrm{C}++$ and MATLAB environments yield trajectories that are nearly consistent with reference trajectories.

\section{Conclusion}

The paper presented the modeling of the intended system concerning the reference frames, coordinates' transformations and equations of motion. This model is built in the form of modules assigned to each process within the guided missile system. Then, it is programmed within the $\mathrm{C}++$ and MATLAB environments. The simulation is conducted with different engagement scenarios and different sources of uncertainties including thrust variation, errors in aerodynamic coefficients and wind velocity effects. The simulation results are validated against reference data with different levels of uncertainty which clarify appropriateness of built 6DOF model for autopilot and guidance laws design with the hardwarein-the-loop (HIL); the objective of next papers.

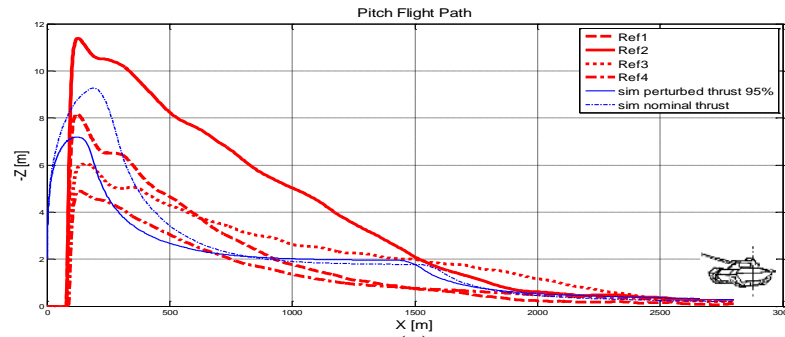

(a)

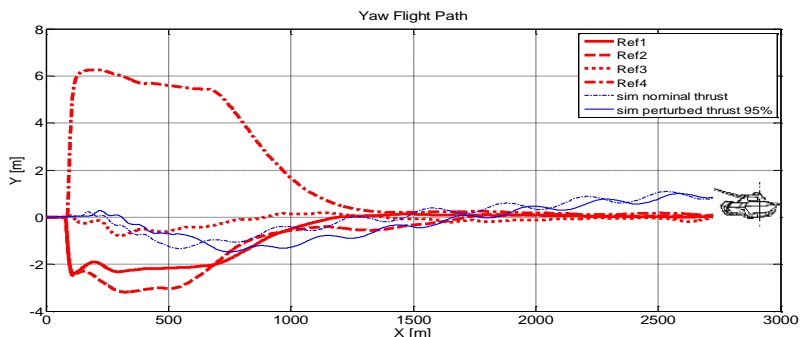

(b)

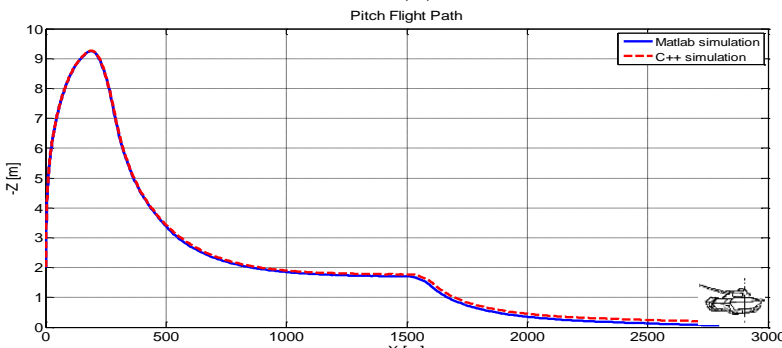

(c)

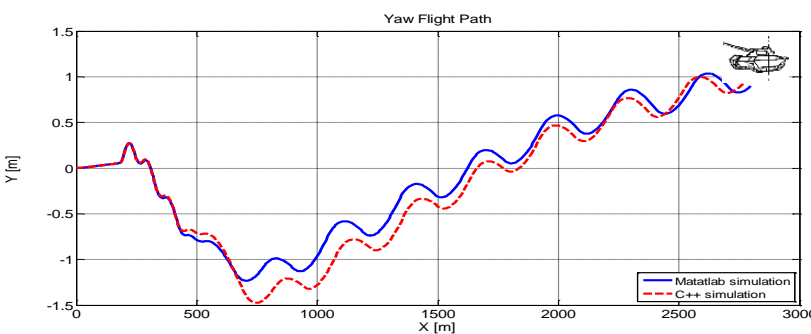

(d)

Fig. 20: Flight simulation and reference trajectories

(a) Pitch Ref. \& MATLAB (b) Yaw Ref. \& MATLAB

(c) Pitch $\mathrm{C}++\&$ MATLAB (d) Yaw $\mathrm{C}++\&$ MATLAB

\section{References}

[1] Abd-Altief, M.A., G.A. El-Sheikh and M.Y. Dogheish, Anti-Tank Guided Missile Performance Enhancement; Part-1: Hardware in the Loop Simulation, 5th ICEENG Conference, GC-9, MTC, Cairo, Egypt, May 16-18, 2006.

[2] Abd El-Latif, M.A., Robust Guidance and Control Algorithms in Presence of Uncertainties, MSc Thesis, Guidance Department, M.T.C., Cairo, Egypt, 2006.

[3] Aly, M.S., Dynamical Analysis of Anti-Tank Missile Systems, Proceedings of the 9th int. AMME conference, 16-18 May, 2000.

[4] Aly, M.S., Linear Model Evaluation of Command Guidance Systems, Proceedings of the 8th Int. conference on aerospace science and aviation technology, pp 1001-1003, 4-6 May, 1999. 
[5] El-Halwagy, Y.Z., M.S. Aly, and M.S. Ghoniemy, Command Guidance System Simulation Airframe Analysis, Proceedings of the 7th Int. conference on aerospace science and aviation technology, 13-15 May, 1997.

[6] El-Sheikh, G.A., Theory of Guidance and Systems, MTC, 2004, 2010.

[7] El-Sheikh, G.A., M.A. Abd-Altief and M.Y. Dogheish, Anti-Tank Guided Missile Performance Enhancement; Part-2: Robust Controller Design, 5th ICEENG Conference, GC-10, MTC, Cairo, Egypt, May 16-18, 2006.

[8] El-Sheikh, G.A., R.A. Elbardeny and N. Maher, Robust Guidance for CLOS System, 12th International Conference on Aerospace Sciences and Aviation Technology (ICASAT12), M.T.C., Cairo, Egypt, May 28-30, 2007 (GUD08-229).

[9] Emil J.E., Test and Evaluation of Tactical Missiles, John Willey \& Sons, (1995).

[10] Etkin B., Dynamics of Flight, Jhon Willey and Sons Inc., 1972.

[11] Garnell P. and East D. J., Guided Weapon Control Systems, 2nd edition, Pergamon press, New York, 1980.

[12] Hertfordshire, S., Guided Weapons, British aircraft corporation, England, May, 1975.

[13] Locke S. and East D.J., Principles Of Guided Missile Design, Van Nostrand Reinhold company Inc., 1955.

[14] Michael selig, Brian fucsz, Flight Simulation, May, 2003.

[15] Oda, A.N., Fuzzy Logic Control and its Application in Guidance Systems, MSc Thesis, Guidance Department, M.T.C., Cairo, Egypt, September, 2005.

[16] Ramadan, N.M., Performance Enhancement of an Anti-Tank Guided Missile Via Advanced Techniques, MSc Thesis, Guidance Department, M.T.C., Cairo, Egypt, September, 2007. 